\title{
Bayesian Networks: A New Approach to Predict Therapeutic Range Achievement of Initial Cyclosporine Blood Concentration After Pediatric Hematopoietic Stem Cell Transplantation
}

\author{
Vincent Leclerc $^{1,2,3} \cdot$ Michel Ducher ${ }^{1,2} \cdot$ Nathalie Bleyzac $^{1,3}$
}

Published online: 5 February 2018

(c) The Author(s) 2018. This article is an open access publication

\begin{abstract}
Background Pediatric hematopoietic stem cell transplantation (HSCT) allows the treatment of numerous diseases, both malignant and non-malignant. Cyclosporine, a narrow therapeutic index drug, is the major immunosuppressant used to prevent graft-versus-host disease (GVHD), but may also cause severe adverse effects in case of overdosing. Objective The objective of this study is to predict the initial cyclosporine residual blood concentration value after pediatric HSCT, and consequently the dose necessary to reach the therapeutic range, using a mathematical individual predictive model.

Methods Clinical and biological data collected from the graft infusion for 2 months after transplantation in 155 pediatric patients undergoing HSCT between 2008 and 2016 were used to generate synthetic data for 1000 subjects which were used to build a Bayesian network model. We compared the characteristics and sensitivity to clinical or biological missing data of this model with four other methods.

Results The tree-augmented Naïve Bayesian network showed the best characteristics, with no missing data (area under the curve of the receiving operator characteristics curve [AUC-ROC] of $0.89 \pm 0.02$ ), $18.9 \pm 2.6 \%$ of patients
\end{abstract}

Michel Ducher

michel.ducher@chu-lyon.fr

1 EMR 3738, Ciblage, Thérapeutique en Oncologie, Faculté de Médecine et de Maïeutique Lyon-Sud Charles Mérieux, Université Claude Bernard Lyon 1, 165 chemin du Grand Revoyet-BP 12, 69921 Oullins Cedex, France

2 Hospices Civils de Lyon, Groupement Hospitalier Nord, Hôpital Pierre Garraud, Service pharmaceutique, 136 rue du Commandant Charcot, 69005 Lyon, France

3 Institute of Pediatric Hematology and Oncology, Place Professeur Joseph Renaut, 69008 Lyon, France misclassified, and positive and negative predictive values of $85.9 \pm 3.4 \%$ and $74.2 \pm 5.1 \%$, respectively, and this trend is found in the synthetic dataset from no to $10 \%$ missing data. The most relevant variables that could influence whether the initial residual cyclosporine concentration is in the therapeutic range are the last dose before measurement and the mean dose before measurement.

Conclusions We developed and cross-validated an online Bayesian network to predict the first cyclosporine concentration after pediatric HSCT. This model allows simulation of different dosing regimens, and enables the best dosing regimen to reach the therapeutic range immediately after transplantation to be found, minimizing the risk of adverse effects and GVHD occurrence.

\section{Key Points}

Bayesian network as a new approach for therapeutic targetting.

Individual prediction of first cyclosporine therapeutic range achievement after pediatric HSCT.

Inclusion of many clinical and biological variables, including inflammation level through CRP value in a cycloporine concentration predictive model after pediatric HSCT.

\section{Introduction}

Hematopoietic stem cell transplantation (HSCT) allows the treatment of numerous diseases in both children and adults, such as hematologic malignancies, non-malignant 
hemopathies, and severe immunodeficiencies $[1,2]$. HSCT became possible in the 1960s, after identification and typing of the human leukocyte antigen (HLA) [3].

In 2015 in France, 5099 HSCTs were performed in 78 centers, and this number has been constantly growing in the last ten years (from 4201 HSCTs in 2006) [4]. Immunosuppressive drugs including cyclosporine are given to prevent graft-versus-host disease (GVHD) [5].

Cyclosporine is a drug with narrow therapeutic index: underdosing may lead to severe GVHD, and overdosing to toxic events or poor graft-versus-leukemia (GVL) effect [5-7]. Thus, therapeutic drug monitoring (TDM) is required and dosage regimens are adapted to reach a target cyclosporine minimal blood concentration value, chosen according to patient's characteristics and disease $[6,8]$.

Many studies have been published on inter-individual pharmacokinetic variability of cyclosporine and Bayesian methods used to optimize dosing regimens. In all cases, population pharmacokinetic models were built, not individual predictive models $[5,6,8]$. To date, no studies have been conducted to predict the achievement of the therapeutic target of the initial cyclosporine blood concentration after HSCT.

In medicine, many methods have been used to predict the value of a target variable, for example, logistic regression in dementia diagnosis [9], Bayesian network models to understand visual field deterioration [10], support vector machines (SVM) for cancer detection [11], and random forest analysis to predict FOLFOX (folinic acid, fluorouracil, and oxaliplatin) responders in colorectal cancer [12]. These new methods seem promising in many fields, but they have scarcely been explored in dosing regimen forecasting [13].

In this study, our objective was to predict whether the initial cyclosporine residual blood concentration after pediatric HSCT will be in the therapeutic range using a mathematical individual predictive model.

\section{Patients and Methods}

The National Committee of Informatics and Liberty approved the current study.

Clinical and biological data were collected from the day of graft infusion to 2 months after transplantation in 155 pediatric patients undergoing HSCT both for malignant and non-malignant diseases between 2008 and 2016. Cyclosporine was given via a 2 -h infusion twice daily, initially $3 \mathrm{mg} / \mathrm{kg}$ for malignant diseases and $5 \mathrm{mg} / \mathrm{kg}$ for non-malignant diseases, starting the day before HSCT. The first measure of cyclosporine trough concentration was performed on whole blood around the third day after the graft infusion $(\mathrm{D}+3)$ (varying between day 0 [D0] and the seventh day after graft infusion [D+7]; median $\mathrm{D}+3$; $83.2 \%$ of measurements taken on $\mathrm{D}+3$ ).

Six patients were excluded from the study as they were given cyclosporine for several weeks before transplantation or took it orally. Fourteen relevant variables that could have possibly influenced cyclosporine concentrations after the first dose post-transplantation were recorded: death, sex, weight, post-transplant day, age, mean dose before measurement, last dose before measurement, HSCT indication, documented infection or not, drugs interacting with cyclosporine, cholestasis, C-reactive protein (CRP) above $15 \mathrm{mg} / \mathrm{L}$, mean hemoglobin, and mean creatinine (see Fig. 1).

All continuous variables were discretized from the literature (initial concentration after transplant, age, hemoglobin, creatinine, and CRP) or from machine learning (other variables such as standard deviations and weight; Netica software version 5.23 [Norsys Software Corp., Vancouver, BC, USA]). The initial cyclosporine residual concentration after transplant was discretized in or out of the therapeutic range, according to the graft indication (malignant or not). The interval retained was $120 \pm 20 \mathrm{mg} /$ $\mathrm{L}$ for malignant disease and $150 \pm 20 \mathrm{mg} / \mathrm{L}$ for non-malignant disease [2]. Age was separated into four classes (from 0 to 2,2 to 8,8 to 14 , and $>14$ years) in accordance with cytochrome P450 (CYP) 3A4 maturation [14-20]. Hemoglobin was discretized into three classes $(<80$, from 80 to 110 , and $>110 \mathrm{~g} / \mathrm{L}$ ) [21]. Serum creatinine was separated into three classes (high, normal, low) according to the patient age and sex. Values considered as normal were $18-26 \mu \mathrm{mol} / \mathrm{L}$ for patients less than 3 years old, 26-44 $\mu \mathrm{mol} / \mathrm{L}$ in patients between 4 and 7 years old, $53-71 \mu \mathrm{mol} / \mathrm{L}$ in patients between 8 and 10 years old, $71-80 \mu \mathrm{mol} / \mathrm{L}$ in females 11 years or older, and $80-106 \mu \mathrm{mol} / \mathrm{L}$ in males aged 13 years and older [22]. Plasma CRP values above $15 \mathrm{mg} / \mathrm{L}$ signaled inflammation $[23,24]$. Cholestasis was defined by a bilirubin plasma concentration above $50 \mu \mathrm{mol} / \mathrm{L}$ [25]. The interacting drugs considered were those that are most used in HSCT: all proton pump inhibitors (PPIs), macrolide antibiotics (including pristinamycine and quinupristine, but excluding spiramycine), all azole antifungals, and calcium channel blockers (nicardipine and amlodipine, but not lacidipine) $[26,27]$.

To generate synthetic data, we used multiple imputation models and, more precisely, data augmentation methods developed by Schafer [28] and Little and Rubin [29]. We generated a dataset of 1000 virtual subjects from the available patients' data $(n=105)$ with Bayesian multiple imputation [30, 31]. We also performed Chi-square tests for categorical variables and $t$ tests for continuous variables to assess if the synthetic data were representative for our database. To assess the performance and the robustness of 
Fig. 1 Bayesian network model to predict the ability of the initial residual blood concentration of cyclosporine after hematopoietic stem cell transplantation to be in the therapeutic range. $C s A$ cyclosporine, $C Y P$ cytochrome P450, $F$ female, HSCT hematopoietic stem cell transplantation, $M$ male

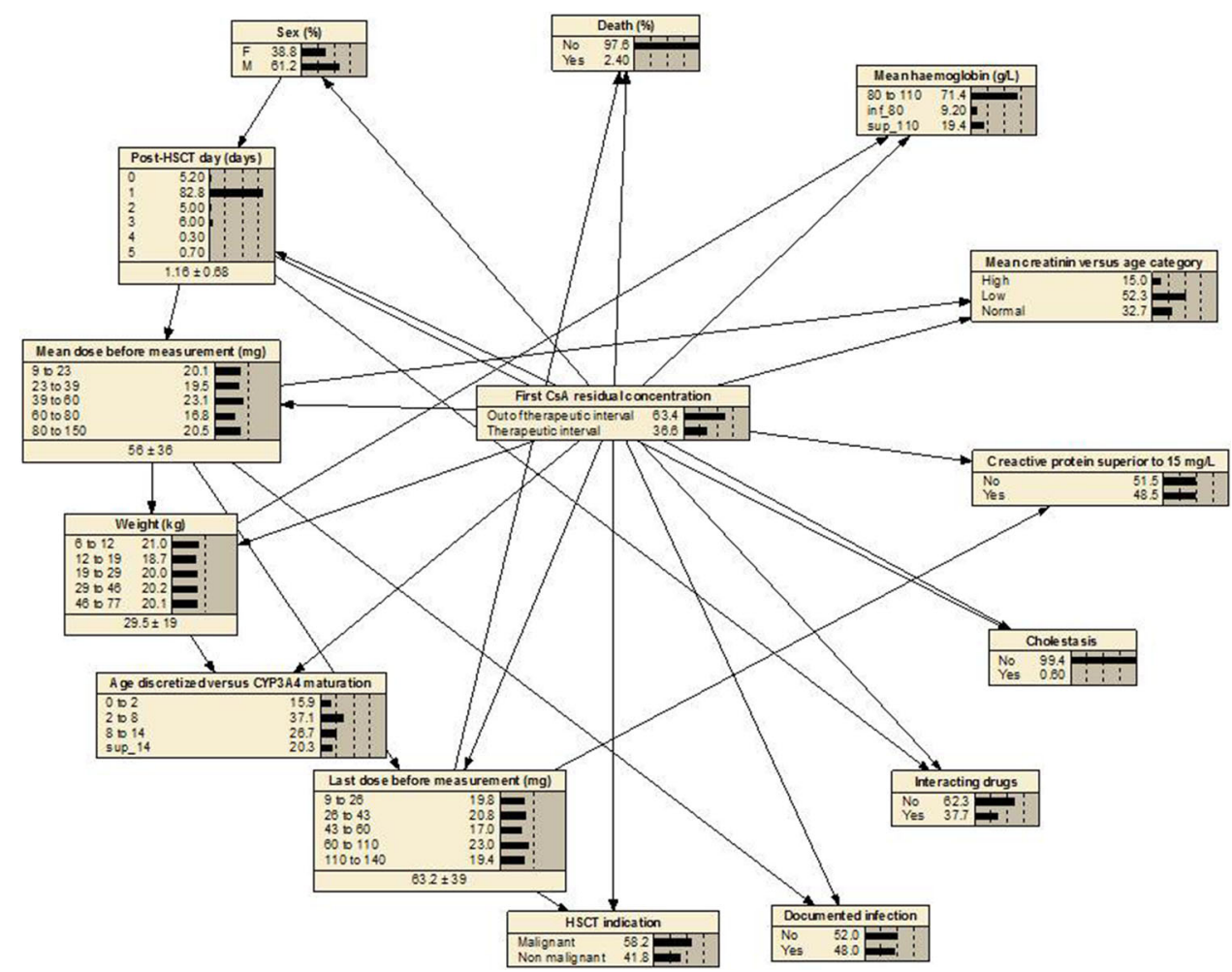

the tree-augmented Naïve (TAN) Bayes algorithm, we compared it to four other methods: logistic regression, Naïve Bayes, SVM, and random forest. The criteria retained for comparison were the area under the curve of the receiving operator characteristics curve (AUC-ROC), the percentage of incorrect patient classification, the positive predictive value, and the negative predictive value, both on training data and after a ten-fold cross-validation.

To test the sensitivity of these methods to missing data, we created different ranges of randomized missing data in the synthetic population: $1,3,5,7,10,15$, and $20 \%$.

The software used for logistic regression, Naïve Bayes network, SVM, and random forest was Orange (version 3.8.1; https://orange.biolab.si/), whereas RapidMiner (version 7.4; RapidMiner, Inc., Boston, MA, USA) was used for TAN Bayes network.

The final TAN Bayesian network model to predict whether the initial CsA residual trough blood concentration after HSCT will be in the therapeutic range was built using Netica software (version 5.23).

To explore the modalities of use of the online model, we performed an analysis of sensitivity to our findings to assess the network ability, as a result of shared information, to compensate for missing variables such as cyclosporine residual concentration, mean dose, mean hemoglobin, mean creatinine, CRP, interacting drugs, cholestasis, documented infection, and weight (Netica software version 5.23).

\section{Results}

Of the 155 patients in total, six were excluded as they were given cyclosporine for several weeks before HSCT or they took cyclosporine orally. Forty-four patients were also excluded from the generation of synthetic data due to one or several missing data at the time of blood sampling for the initial determination of the cyclosporine residual blood concentration following HSCT. A final total of 105 patients without missing data at this time were retained (see Fig. 2). The characteristics of the 105 patients are summarized in Table 1.

Of these 105 patients, 37 had initial cyclosporine concentration values within the therapeutic interval, and 41 and 27 had sub-therapeutic and over-therapeutic cyclosporine residual concentration values, respectively. The HSCT indication was acute lymphoid leukemia (34\%), inborn error (18\%), immune deficiency (16\%), severe aplastic anemia (9\%), acute myeloid leukemia (6\%), lymphoma (6\%), myelodysplastic syndrome (6\%), severe hemoglobinopathies (3\%), and chronic myeloid leukemia (2\%). Only one patient presented a cholestasis (total bilirubin over $50 \mu \mathrm{mol} / \mathrm{L}$ ) at this time. Thirty-eight patients took interacting medications: two took azole antifungals, two macrolides, 32 PPIs, one a calcium channel blocker, and one both a PPI and a calcium channel blocker. The youngest patient was 5 months old, the oldest was 21 years old, and the median age was 7 years. 
Fig. 2 Flow chart. CsA cyclosporine, $H S C T$ hematopoietic stem cell transplantation

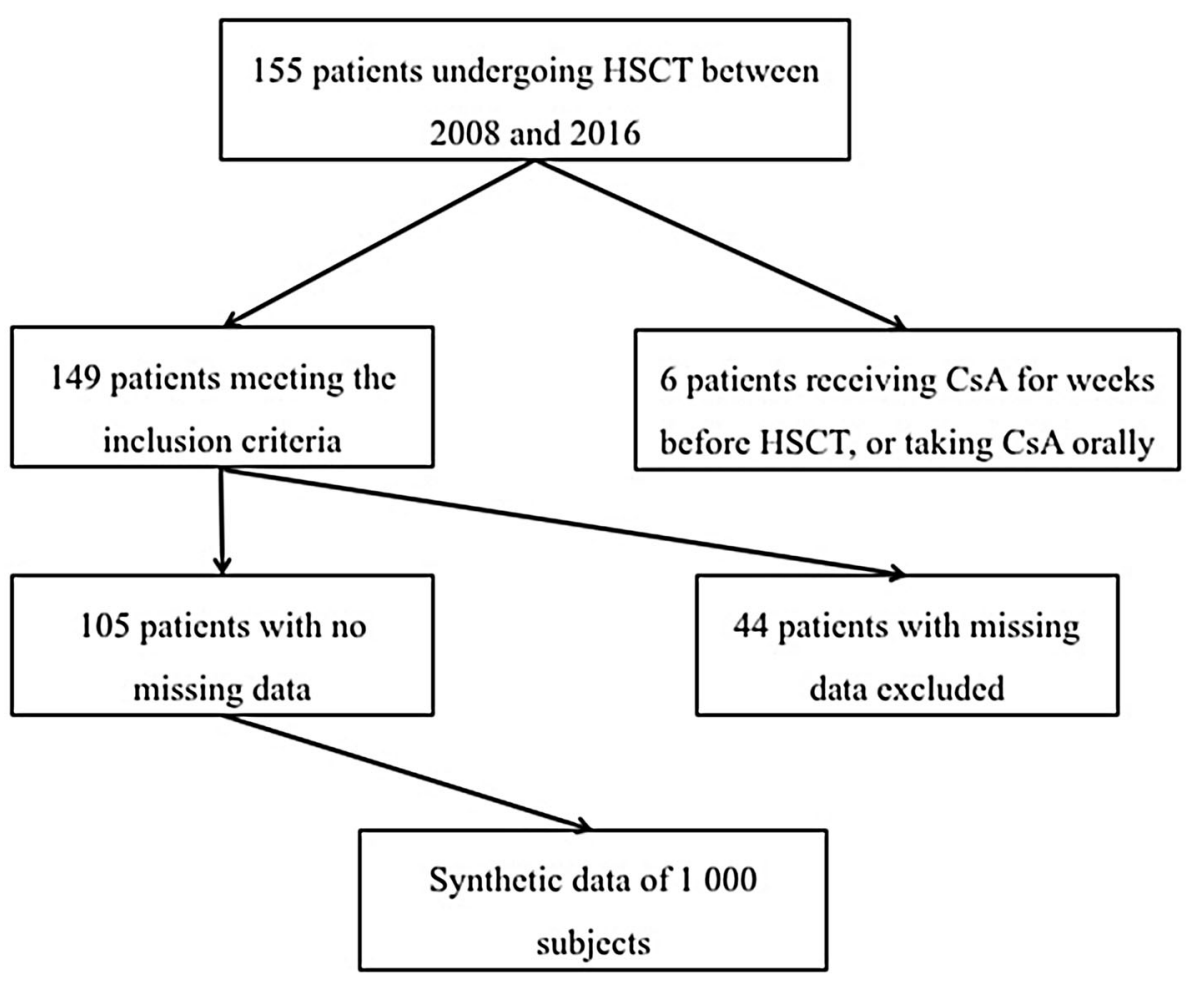

\subsection{Modelling Procedure}

We assessed that the synthetic dataset was representative for our database by a visual check of the probability densities distribution, and by comparing the variables of interest (Table 1).

Sensitivity for missing data of the synthetic dataset was evaluated for the five methods and illustrated with AUCROC evolution after a ten-fold cross-validation. With no missing data, the TAN Bayesian network was the best method (AUC-ROC 0.893), and SVM was the worst (AUC-ROC 0.673) (Table 2). The TAN Bayesian network was the least robust (AUC-ROC from 0.893 with no missing data to 0.7 with $20 \%$ of missing data, resulting in a loss of 0.193 points), but presented the best characteristics since $10 \%$ of missing data in the synthetic dataset (AUCROC $0.785,25.8 \%$ incorrectly classified patients, and a predictive positive value of 0.74 ).

\subsection{Validation of the Predictive Model}

The final predictive model for the ability of the initial cyclosporine trough blood concentration after HSCT to be in the therapeutic range, after a tenfold cross-validation, presented good validation characteristics with an AUCROC of $0.89 \pm 0.02$ (see Fig. 3), $18.9 \pm 2.6 \%$ of incorrectly classified patients, a positive predictive value of $85.9 \pm 3.4 \%$ and a negative predictive value of $74.2 \pm 5.1 \%$.

\subsection{Sensitivity to Missing Data of the Final Tree- Augmented Naïve (TAN) Bayesian Network}

The results of the analysis of sensitivity to findings that was performed on the synthetic population are shown in Fig. 4. The most relevant variables that could influence whether the initial residual blood cyclosporine concentration was in the therapeutic range were the last dose before measurement, the mean dose before measurement, and the day of blood sampling after HSCT. Some variables were required for the prediction and could not be derived from other ones. This was especially the case for interacting drugs, mean hemoglobin, and mean creatinine, whereas a missing weight could adequately be replaced by information on the last administered dose, mean dose before measurement, age versus CYP3A4 maturation, mean hemoglobin, and mean creatinine versus age category.

The final model can be found at https://www.hed.cc/?s= HSCTCsApredictor\&t=HSCT CsA predictor.

\section{Discussion}

In our pediatric population undergoing HSCT who were receiving cyclosporine to prevent GVHD, TAN Bayesian network was the best method to predict whether the initial cyclosporine concentration after HSCT will be in the therapeutic range. The therapeutic range of the cyclosporine blood concentration rather than cyclosporine blood 
Table 1 Population characteristics at initial time blood sampling for cyclosporine residual concentration after hematopoietic stem cell transplantation

\begin{tabular}{|c|c|c|c|c|}
\hline Variable & $\begin{array}{l}\text { Available patients } \\
(n=105)\end{array}$ & $\begin{array}{l}\text { Virtual subjects } \\
(n=1000)\end{array}$ & $\begin{array}{l}\text { Chi-square test } \\
(\alpha=0.05)\end{array}$ & $\begin{array}{l}t \text { test } \\
(\alpha=0.05)\end{array}$ \\
\hline \multicolumn{5}{|l|}{$\operatorname{Sex}(\%)$} \\
\hline Male & 63.8 & 61.2 & NS & \\
\hline Female & 36.2 & 38.8 & NS & \\
\hline \multicolumn{5}{|l|}{ Age (years) } \\
\hline $0-2$ & 15.2 & 15.9 & NS & \\
\hline $2-8$ & 37.1 & 37.1 & NS & \\
\hline $8-14$ & 25.7 & 26.7 & NS & \\
\hline$>14$ & 21.9 & 20.3 & NS & \\
\hline Weight (kg) & $30.1 \pm 19$ & $29.5 \pm 19$ & & NS \\
\hline \multicolumn{5}{|c|}{ Initial CsA residual trough blood concentration after HSCT (\%) } \\
\hline Within therapeutic interval & 35.2 & 36.6 & NS & \\
\hline Out of therapeutic interval & 64.8 & 63.4 & NS & \\
\hline \multicolumn{5}{|l|}{ HSCT indication (\%) } \\
\hline Malignant & 56.2 & 58.2 & NS & \\
\hline Non-malignant & 43.8 & 41.8 & NS & \\
\hline \multicolumn{5}{|l|}{ Mean hemoglobin (g/L) } \\
\hline$<80$ & 9.5 & 9.2 & NS & \\
\hline $80-110$ & 71.4 & 71.4 & NS & \\
\hline$>110$ & 19.1 & 19.4 & NS & \\
\hline \multicolumn{5}{|c|}{ Mean creatinine versus age category $(\mu \mathrm{mol} / \mathrm{L})$} \\
\hline Low & 54.3 & 52.3 & NS & \\
\hline Normal & 31.4 & 32.7 & NS & \\
\hline High & 14.3 & 15 & NS & \\
\hline \multicolumn{5}{|l|}{$\mathrm{CRP}>15$ mg/L $(\%)$} \\
\hline Yes & 48.6 & 48.5 & NS & \\
\hline No & 51.4 & 51.5 & NS & \\
\hline $\begin{array}{l}\text { Mean dose before measurement } \\
(\mathrm{mg})\end{array}$ & $56.9 \pm 37$ & $56 \pm 36$ & & NS \\
\hline Last dose before measurement (mg) & $63.9 \pm 39$ & $63.2 \pm 39$ & & NS \\
\hline \multicolumn{5}{|l|}{ Cholestasis (\%) } \\
\hline Yes & 0.9 & 0.6 & NS & \\
\hline No & 99.1 & 99.4 & NS & \\
\hline \multicolumn{5}{|l|}{ Interacting drug $(\%)$} \\
\hline Yes & 36.2 & 37.7 & NS & \\
\hline No & 63.8 & 62.3 & NS & \\
\hline \multicolumn{5}{|l|}{ Documented infection $(\%)$} \\
\hline Yes & 45.7 & 48 & NS & \\
\hline No & 53.3 & 52 & NS & \\
\hline \multicolumn{5}{|l|}{ Death (\%) } \\
\hline Yes & 2.9 & 2.4 & NS & \\
\hline No & 97.1 & 97.6 & NS & \\
\hline
\end{tabular}

CRP C-reactive protein, CsA cyclosporine, HSCT hematopoietic stem cell transplantation, NS not significant

concentration itself was chosen as the endpoint because it seemed the most clinically relevant and simplest to handle for clinicians while using an online Bayesian network. This tool will allow them to find the best cyclosporine dosing regimen to reach the therapeutic interval right after transplantation, using a simple method, provided that further external validation is satisfactory. 
Table 2 Characteristics of the five methods with no missing data after ten-fold cross-validation

\begin{tabular}{llllll}
\hline & Logistic regression & Naïve Bayes & Tree-augmented Naïve Bayes & Support vector machines & Random forest \\
\hline AUC-ROC & 0.84 & 0.78 & 0.89 & 0.67 & 0.85 \\
\% incorrect classification & 23.6 & 28.5 & 18.9 & 32.1 & 23.2 \\
Positive predictive value & 0.70 & 0.64 & 0.86 & 0.55 & 0.70 \\
Negative predictive value & 0.79 & 0.75 & 0.74 & 0.78 & 0.80 \\
\hline
\end{tabular}

$A U C-R O C$ area under the curve of the receiving operator characteristics curve

Fig. 3 Receiving operator characteristics curve of the final Bayesian network

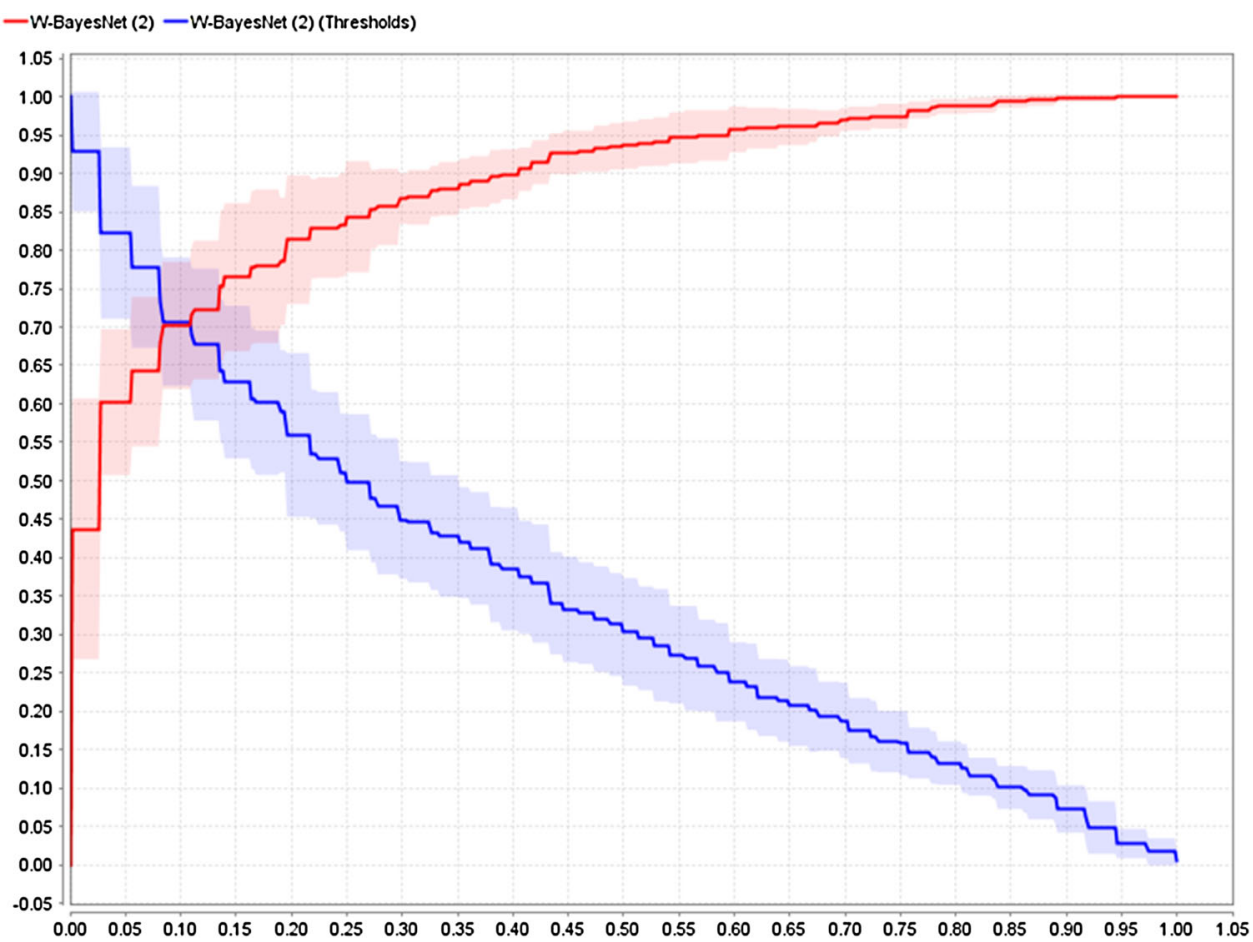

Fig. 4 Relative percentage of missing information compensated by the final network. CRP C-reactive protein, $C s A$ cyclosporine, HSCT hematopoietic stem cell transplantation

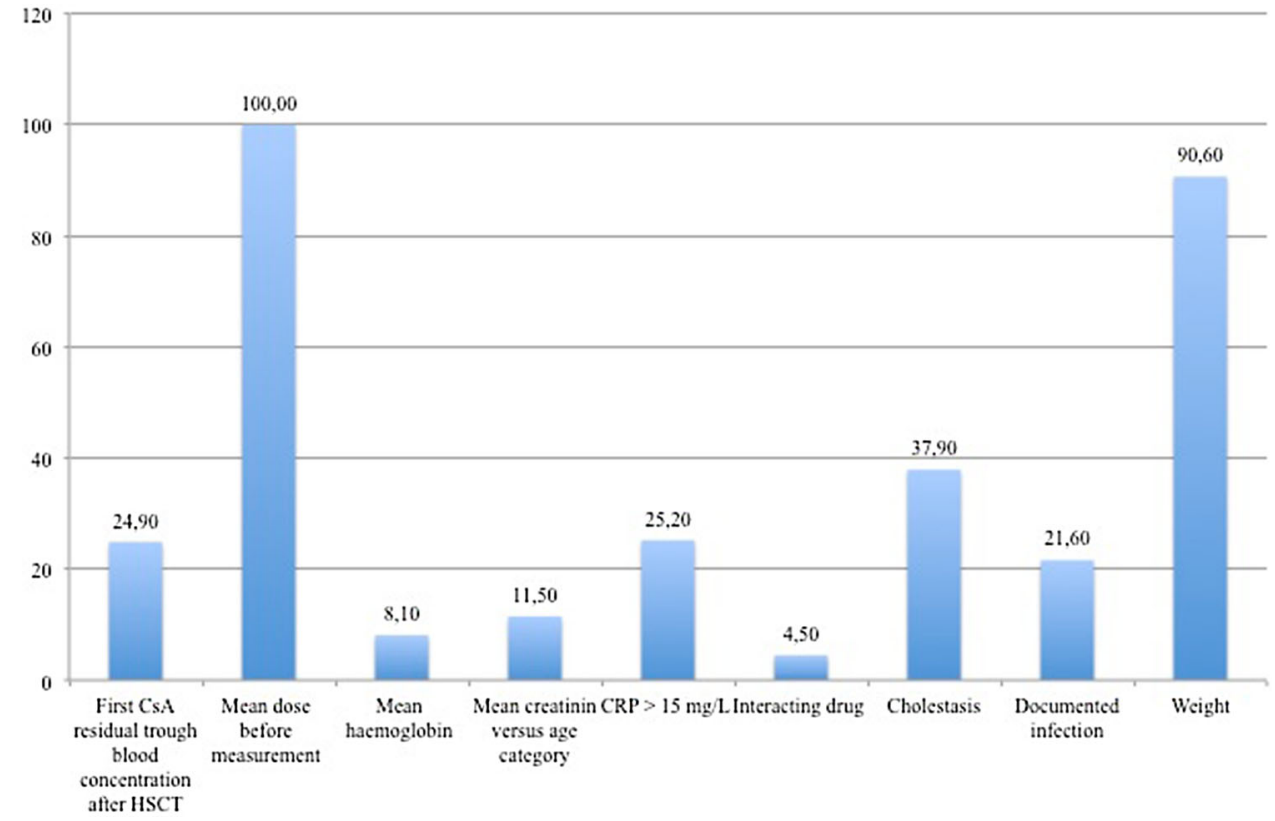


Many classification and prediction methods are used in medicine, and we chose to limit our study to five that are widely used: one parametric (logistic regression) and four non-parametric (Naïve Bayesian network, TAN Bayesian network, SVM, and random forest).

Belief networks are directed acyclic graphs that represent a probabilistic structure. There is a direct relationship between the graphical model and a particular probability distribution form. They consist of vertices (or nodes) and directed edges (arrows). Each edge points from one node (called the parent node) to another node (the child node). In a belief network each node is used to represent a random variable, and each directed edge represents an immediate dependence or direct influence. We used a learning algorithm to create a TAN Bayes graph structure in which a single-class variable has no parents and all other variables have the class as a parent and at most one other attribute as a parent. The probability tables are filled out using Expectation Maximization, as previously described in Sansot et al. [13].

Logistic regression, a widely used method in biology and medicine, allows the association between several variables to be quantified by estimating a probability. This technique takes confounding factors into account but requires the measures to be of good quality and may be disturbed by interactions between variables. It also cannot deal with missing data relating to continuous variables [32, 33].

SVM are learning algorithms with the ability to separate several variables into classes, with an optimal hyper-plane maximizing the margin. SVM are well-suited to work involving high dimensional data, and have robust performances when dealing with sparse and noisy data [34].

Random forest is a method based on the generation of multiple trees by bootstrapping. For each node of each tree, the best predictor is chosen. The final result given by random forest is the mean of all the individual trees [35].

To compare these methods, we had to ensure optimal conditions that avoided variance homogeneity issues, lack of statistical power, and sensitivity towards missing data, which can influence parametric methods. That is why an approach based on synthetic data was preferred. After cross-validation, TAN Bayesian network was the best method according to the comparison of the AUC-ROC curves and its very good positive predictive value.

Moreover, the synthetic data approach allowed us to test the influence of missing data, taking of statistical power issues into account. The trend in which TAN Bayesian network is the best method was also verified even in the context of $10 \%$ missing data.

Our approach testing the sensitivity of our method with regards to missing data seems very important as data in clinical practice are often incomplete because of real-life conditions.
The TAN Bayesian network, in addition to its other positive characteristics, is also easier to use and understand by clinicians than other methods such as random forest. Indeed, its representation as a directed acyclic graph allows a quick and simple answer to a question, which is consistent with what is required in clinical practice.

The final TAN Bayesian network shows, as it did in the simulation approach, good validation characteristics.

This model is intended to be online, and thus it has to respond to two major concerns: sensitivity for missing data and external validation. The sensitivity analysis performed informs us on the importance of the information provided by some variables to the network.

Information on variables which cannot be compensated for by other variables has to be filled in the online network; these indispensable variables will be listed in the user manual of the online model. This model seems very interesting for clinicians as it would allow them to predict whether patients will reach the therapeutic range of concentrations immediately after HSCT or not, and consequently a priori change the cyclosporine dose regimen. However, an external multicentric validation is warranted for this model before its use in clinical practice.

Over the past 50 years HSCT has been a huge progress in medicine, and consequently TDM of immunosuppressants became a great concern. As cyclosporine is the main immunosuppressant used after HSCT, many researchers have worked on the link between the concentration of cyclosporine and the onset of GVHD [36-39]. Malard et al. [39] have shown that trough blood cyclosporine concentrations during the first week after HSCT were correlated with the onset of grade III-IV acute GVHD, but no increase in overall survival was found. However, in this study, the cyclosporine target concentrations were high, inhibiting a possible GVL effect, and exposing patients to cyclosporine adverse effects [39]. Martin et al. [2] also found this trend in a pediatric population. In this way, our model could be useful, allowing the therapeutic interval to be reached early after HSCT, and it could be evaluated for its use in improving the onset of moderate acute GVHD in malignant diseases (optimizing the GVL effect) or decreasing the rate of severe GVHD in non-malignant diseases.

The first studies only took into account the serum creatinine and the trough blood cyclosporine concentration when adapting the cyclosporine dosing regimen [37, 38], but complex models taking into account many covariates such as hemoglobin, hematocrit, hepatic functions (through bilirubin, alanine aminotransferase [ALAT], aspartate aminotransferase [ASAT], albumin), and creatinine have been developed in more recent years [40]. However, to date, our model is the first to include so many variables at the same time to predict the achievement of the 
cyclosporine therapeutic index soon after HSCT. Moreover, the role of inflammation on cytochromes, notably CYP3A4, and subsequent inhibition of metabolism of drugs such as cyclosporine is well-known [41]. Inflammatory episodes are frequently associated with HSCT, for example post-transplant infections. Consequently, the inclusion of inflammation parameters in the model is relevant. Nevertheless, our study is the first to take some of these parameters into account.

\subsection{Limitations}

It should be noted that some other variables may influence cyclosporine trough blood concentrations, but these are not available nor considered in clinical practice, and thus this model does not include them.

Cyclosporine trough blood concentrations present a high inter- and intra-individual variability and their evolution over the treatment period is a complex phenomenon. The possibility to consider many variables at the same time means that there is real hope that Bayesian networks will aid establishment of useful models for clinicians.

This model is interesting but it concerns only the initial cyclosporine concentration after pediatric HSCT. External validation on another cohort of patients will be necessary before it can be used in clinical practice.

Another limitation is the therapeutic range of cyclosporine considered in our study. Indeed, therapeutic cyclosporine blood concentration target ranges have been set only according to the graft indication, since the concentration of interest was the first one after transplantation. If a longer period after HSCT is considered, occurrence of GVHD and other target cyclosporine concentration values will have to be taken into account. Moreover, Jacobson et al. [42] have shown that the number of days posttransplant influences cyclosporine pharmacokinetics, which is why it would probably be interesting to develop a model covering a larger duration after HSCT in order to take into account the intra-individual variability of cyclosporine pharmacokinetics.

\section{Conclusion}

In this study we have developed and validated an online Bayesian network to assess the ability of the initial cyclosporine blood concentration to be in the therapeutic interval after pediatric HSCT. This model allows the simulation of different dosing regimens, finding the best to enable the right cyclosporine residual concentration to be reached immediately after transplantation, minimizing the risk of adverse effects and GVHD occurrence. The characteristics of this model are satisfactory but external multicentric validation is needed before its use in clinical practice.

Funding Vincent Leclerc, Michel Ducher, and Nathalie Bleyzac have no funding to report.

\section{Compliance with Ethical Standards}

Conflict of interest Vincent Leclerc, Michel Ducher, and Nathalie Bleyzac declare no conflicts of interest.

Open Access This article is distributed under the terms of the Creative Commons Attribution-NonCommercial 4.0 International License (http://creativecommons.org/licenses/by-nc/4.0/), which permits any noncommercial use, distribution, and reproduction in any medium, provided you give appropriate credit to the original author(s) and the source, provide a link to the Creative Commons license, and indicate if changes were made.

\section{References}

1. Bleyzac N. The use of pharmacokinetic models in paediatric onco-haematology: effects on clinical outcome through the examples of busulfan and cyclosporine. Fundam Clin Pharmacol. 2008;22:605-8.

2. Martin P, Bleyzac N, Souillet G, Galambrun C, Bertrand Y, Maire PH, et al. Relationship between CsA trough blood concentration and severity of acute graft-versus-host disease after paediatric stem cell transplantation from matched-sibling or unrelated donors. Bone Marrow Transplant. 2003;32:777-84.

3. Copelan EA. Hematopoietic stem-cell transplantation. N Engl J Med. 2006;354:1813-26.

4. Agence de la biomédecine - Le rapport annuel médical et scientifique. 2015. https://www.agence-biomedecine.fr/annexes/ bilan2015/donnees/cellules/04-national/synthese.htm\#. Accessed 19 April 2017.

5. Bleyzac N, Cuzzubbo D, Renard C, Garnier N, Dubois V, Domenech $\mathrm{C}$, et al. Improved outcome of children transplanted for high-risk leukemia by using a new strategy of cyclosporinebased GVHD prophylaxis. Bone Marrow Transplant. 2016;51:698-704.

6. Shaw LM. Advances in cyclosporine pharmacology, measurement, and therapeutic monitoring. Clin Chem. 1989;35:1299-308.

7. Dunn CJ, Wagstaff AJ, Perry CM, Plosker GL, Goa KL. Cyclosporin: an updated review of the pharmacokinetic properties, clinical efficacy and tolerability of a microemulsion-based formulation (neoral)1 in organ transplantation. Drugs. 2001;61:1957-2016.

8. Kahan BD. Therapeutic drug monitoring of cyclosporine: 20 years of progress. Transplant Proc. 2004;36:378S-91S.

9. Mazzocco T, Hussain A. Novel logistic regression models to aid the diagnosis of dementia. Expert Syst Appl. 2012;39:3356-61.

10. Tucker A, Vinciotti V, Liu X, Garway-Heath D. A spatio-temporal Bayesian network classifier for understanding visual field deterioration. Artif Intell Med. 2005;34:163-77.

11. Wang H, Huang G. Application of support vector machine in cancer diagnosis. Med Oncol. 2011;28(Suppl 1):S613-8.

12. Tsuji S, Midorikawa Y, Takahashi T, Yagi K, Takayama T, Yoshida K, et al. Potential responders to FOLFOX therapy for colorectal cancer by Random Forests analysis. $\mathrm{Br} \mathrm{J}$ Cancer. 2012;106:126-32. 
13. Sansot C, Kalbacher E, Lemoine S, Bourguignon L, Fauvel J-P, Ducher M. A Bayesian model to describe factors influencing trough levels of vancomycin in hemodialysis patients. Nephron. 2015;131:131-7.

14. Hesselink DA, van Schaik RHN, Nauta J, van Gelder T. A drug transporter for all ages? ABCB1 and the developmental pharmacogenetics of cyclosporine. Pharmacogenomics. 2008;9:783-9.

15. de Wildt SN, Kearns GL, Leeder JS, van den Anker JN. Cytochrome P450 3A: ontogeny and drug disposition. Clin Pharmacokinet. 1999;37:485-505.

16. Kanamori M, Takahashi H, Echizen H. Developmental changes in the liver weight- and body weight-normalized clearance of theophylline, phenytoin and cyclosporine in children. Int J Clin Pharmacol Ther. 2002;40:485-92.

17. Hakkola J, Tanaka E, Pelkonen O. Developmental expression of cytochrome P450 enzymes in human liver. Pharmacol Toxicol. 1998;82:209-17.

18. Fanta S, Jonsson S, Backman JT, Karlsson MO, Hoppu K. Developmental pharmacokinetics of ciclosporin-a population pharmacokinetic study in paediatric renal transplant candidates. Br J Clin Pharmacol. 2007;64:772-84.

19. Fakhoury M, Litalien C, Medard Y, Cavé H, Ezzahir N, Peuchmaur M, et al. Localization and mRNA expression of CYP3A and P-glycoprotein in human duodenum as a function of age. Drug Metab Dispos Biol Fate Chem. 2005;33:1603-7.

20. Bouillon-Pichault M, Jullien V, Bazzoli C, Pons G, Tod M. Pharmacokinetic design optimization in children and estimation of maturation parameters: example of cytochrome P450 3A4. J Pharmacokinet Pharmacodyn. 2011;38:25-40.

21. Sullivan KM, Mei Z, Grummer-Strawn L, Parvanta I. Haemoglobin adjustments to define anaemia. Trop Med Int Health. 2008;13:1267-71.

22. Atiyeh BA, Dabbagh SS, Gruskin AB. Evaluation of renal function during childhood. Pediatr Rev. 1996;17:175-80.

23. McNeer JL, Kletzel M, Rademaker A, Alford K, O'Day K, Schaefer C, et al. Early elevation of C-reactive protein correlates with severe infection and nonrelapse mortality in children undergoing allogeneic stem cell transplantation. Biol Blood Marrow Transplant. 2010;16:350-7.

24. Fuji S, Kim S-W, Fukuda T, Mori S, Yamasaki S, Morita-Hoshi $\mathrm{Y}$, et al. Preengraftment serum C-reactive protein (CRP) value may predict acute graft-versus-host disease and nonrelapse mortality after allogeneic hematopoietic stem cell transplantation. Biol Blood Marrow Transplant. 2008;14:510-7.

25. Wilhelm AJ, de Graaf P, Veldkamp AI, Janssen JJWM, Huijgens PC, Swart EL. Population pharmacokinetics of ciclosporin in haematopoietic allogeneic stem cell transplantation with emphasis on limited sampling strategy. Br J Clin Pharmacol. 2012;73:553-63.

26. Bernard E, Goutelle S, Bertrand Y, Bleyzac N. Pharmacokinetic drug-drug interaction of calcium channel blockers with cyclosporine in hematopoietic stem cell transplant children. Ann Pharmacother. 2014;48:1580-4.
27. Bernard E, Mialou V, Dony A, Garnier N, Renard C, Bleyzac N. Lacidipine efficacy and safety for high blood pressure treatment in pediatric oncohematology [in French]. Arch Pediatr. 2014;21:1101-5.

28. Schafer JL. Analysis of incomplete multivariate data. Boca Raton: CRC Press; 1997.

29. Little RJA, Rubin DB. Statistical analysis with missing data. Hoboken: Wiley; 2014.

30. Raghunathan TE, Reiter JP, Rubin DB. Multiple imputation for statistical disclosure limitation. J Off Stat. 2003;19:1-16.

31. Reiter JP. Satisfying disclosure restrictions with synthetic data sets. J Off Stat. 2002;18:531-44.

32. Tolles J, Meurer WJ. Logistic regression: relating patient characteristics to outcomes. JAMA. 2016;316:533-4.

33. Meurer WJ, Tolles J. Logistic regression diagnostics: understanding how well a model predicts outcomes. JAMA. 2017;317:1068-9.

34. Furey TS, Cristianini N, Duffy N, Bednarski DW, Schummer M, Haussler D. Support vector machine classification and validation of cancer tissue samples using microarray expression data. Bioinformatics. 2000;16:906-14.

35. Lombardo F, Obach RS, Dicapua FM, Bakken GA, Lu J, Potter $\mathrm{DM}$, et al. A hybrid mixture discriminant analysis-random forest computational model for the prediction of volume of distribution of drugs in human. J Med Chem. 2006;49:2262-7.

36. Yee GC, Self SG, McGuire TR, Carlin J, Sanders JE, Deeg HJ. Serum cyclosporine concentration and risk of acute graft-versushost disease after allogeneic marrow transplantation. $\mathrm{N}$ Engl $\mathrm{J}$ Med. 1988;319:65-70.

37. Stockschlaeder M, Storb R, Pepe M, Longton G, McDonald G, Anasetti C, et al. A pilot study of low-dose cyclosporin for graftversus-host prophylaxis in marrow transplantation. Br J Haematol. 1992;80:49-54.

38. Ghalie R, Fitzsimmons WE, Weinstein A, Manson S, Kaizer H. Cyclosporine monitoring improves graft-versus-host disease prophylaxis after bone marrow transplantation. Ann Pharmacother. 1994;28:379-83.

39. Malard F, Szydlo RM, Brissot E, Chevallier P, Guillaume T, Delaunay $\mathbf{J}$, et al. Impact of cyclosporine-A concentration on the incidence of severe acute graft-versus-host disease after allogeneic stem cell transplantation. Biol Blood Marrow Transplant. 2010;16:28-34.

40. Woillard J-B, Lebreton V, Neely M, Turlure P, Girault S, Debord $\mathrm{J}$, et al. Pharmacokinetic tools for the dose adjustment of ciclosporin in haematopoietic stem cell transplant patients. Br J Clin Pharmacol. 2014;78:836-46.

41. Schultz KR, Nevill TJ, Balshaw RF, Toze CL, Corr T, Currie CJ, et al. Effect of gastrointestinal inflammation and age on the pharmacokinetics of oral microemulsion cyclosporin A in the first month after bone marrow transplantation. Bone Marrow Transplant. 2000;26:545-51.

42. Jacobson PA, Ng J, Green KGE, Rogosheske J, Brundage R. Posttransplant day significantly influences pharmacokinetics of cyclosporine after hematopoietic stem cell transplantation. Biol Blood Marrow Transplant. 2003;9:304-11. 